\title{
Some high-frequency gravitational waves related to exact radiative spacetimes
}

\author{
J. Podolský and O. Svítek*
}

October 24, 2018

\begin{abstract}
A formalism is introduced which may describe both standard linearized waves and gravitational waves in Isaacson's high-frequency limit. After emphasizing main differences between the two approximation techniques we generalize the Isaacson method to non-vacuum spacetimes. Then we present three large explicit classes of solutions for high-frequency gravitational waves in particular backgrounds. These involve non-expanding (plane, spherical or hyperboloidal), cylindrical, and expanding (spherical) waves propagating in various universes which may contain a cosmological constant and electromagnetic field. Relations of high-frequency gravitational perturbations of these types to corresponding exact radiative spacetimes are described.
\end{abstract}

PACS: 04.30.-w; 04.25.-g; 04.20.Jb

Key words: gravitational waves, high-frequency limit, exact solutions

\section{Introduction}

In classic work [1] Isaacson presented a perturbation method which enables one to study properties of high-frequency gravitational waves, together with their influence on the cosmological background in which they propagate. It is this non-linear "back-reaction" effect on curvature of the background spacetime which distinguishes the high-frequency approximation scheme from other perturbation methods such as the standard Einstein's linearization of gravitational field in flat space [2,3] or multipole expansions [4] that were developed to describe radiation from realistic astrophysical sources.

The high-frequency perturbations were originally considered by Wheeler [5] and then applied to investigation of gravitational geons by Brill and Hartle [6]. Isaacson's systematic study [1] stimulated further works in which his treatment was developed and also re-formulated in various formalisms. Choquet-Bruhat [7,8, analyzed high-frequency gravitational radiation using a generalized WKB "two-timing" method. Averaged Lagrangian technique which leads to Isaacson's results with less calculation was introduced by MacCallum and Taub 9, 10. Comparison of these approaches, and clarification of assumptions that have to be made in order to provide a consistent high-frequency approximation limit was also given by Araujo 11, 12. Elster [13. proposed an alternative method that is based on expanding null-tetrad components of the Weyl tensor. Recently, Burnett developed a weak limit approach [14] in which the high-frequency limit

\footnotetext{
${ }^{*}$ Institute of Theoretical Physics, Charles University in Prague, V Holešovičkách 2, 18000 Prague 8, Czech Republic
} 
can be introduced and studied in a mathematically rigorous way. These general methods have been, of course, applied to study explicit particular examples of high-frequency gravitational waves, see e.g. [1, 8, 9, 15, 16.

On the other hand, many exact solutions of Einstein's equations are known which represent gravitational radiation. Among the most important classes are planar $p p$-waves [17, 18, which belong to a large family of non-expanding radiative spacetimes [19 20], cylindrical Einstein-Rosen waves [21, expanding "spherical" waves of the Robinson-Trautman type [22, 23, spacetimes with boost-rotation symmetry representing radiation generated by uniformly accelerated sources 24, 25, 26], cosmological models of the Gowdy type [27, and others - for comprehensive reviews containing also a number of references see, e.g., [28, 30, 31, 29, 32].

However, there are only several works in which relation between exact gravitational waves and those obtained by perturbations of non-flat backgrounds has been explicitly investigated and clarified, see e.g. 10,33, 16. The purpose of our contribution is to help to fill this "gap".

We first briefly summarize and generalize the Isaacson approach [1] to admit non-vacuum backgrounds, the cosmological constant $\Lambda$ in particular. Modification of Isaacson's formalism allows us to incorporate also standard linearized gravitational waves into the common formalism. Then, in section 3 we study properties of high-frequency gravitational waves in specific classes of spacetimes with special algebraic or geometric structure. In particular, we focus on waves which propagate in backgrounds with $\Lambda \neq 0$. This is motivated not only theoretically but also by recent observations 34 which seem to indicate that (effective) positive cosmological constant played a fundamental role in the early universe, but it is also important for its present and future dynamics.

\section{High-frequency approximation versus standard linearization}

Let us assume a formal decomposition of the spacetime metric $g_{\mu \nu}$ into the background metric $\gamma_{\mu \nu}$ and its perturbation $h_{\mu \nu}$

$$
g_{\mu \nu}=\gamma_{\mu \nu}+\varepsilon h_{\mu \nu}
$$

where, in a suitable coordinate system, $\gamma_{\mu \nu}=O(1)$ and $h_{\mu \nu}=O(\epsilon)$ [by definition, $f=O\left(\epsilon^{n}\right)$ if there exists a constant $C>0$ such that $|f|<C \epsilon^{n}$ as $\left.\epsilon \rightarrow 0\right]$. The two distinct non-negative dimensionless parameters $\varepsilon$ and $\epsilon$ have the following meaning: $\varepsilon$ is the usual amplitude parameter of weak gravitational perturbations whereas the frequency parameter $\epsilon$ denotes the possible highfrequency character of radiation described by $h_{\mu \nu}$. To be more specific, the parameter $\varepsilon \ll 1$ characterizes (for $\epsilon=1$ ) the amplitude of linearized gravitational waves in the ordinary weak field limit of Einstein's equations. The second independent parameter $\epsilon=\lambda / L$ represents, on the other hand, the ratio of a typical wavelength $\lambda$ of gravitational waves and the scale $L$ on which the background curvature changes significantly. Isaacson's high-frequency approximation [1] arises when $\lambda \ll L$, i.e. $\epsilon \ll 1$ (and $\varepsilon=1$ ). Since $L$ can be considered to have a finite value of order unity, we may write $O(\epsilon)=O(\lambda)$.

To derive the dynamical field equations we start with the order-of-magnitude estimates which indicate how fast the metric components vary. Symbolically, the derivatives are of the order $\partial \gamma \sim \gamma / L, \partial h \sim h / \lambda$, so that the following formulas

$$
\begin{aligned}
& \gamma_{\mu \nu}=O(1), \quad h_{\mu \nu}=O(\epsilon), \\
& \gamma_{\mu \nu, \alpha}=O(1), \quad h_{\mu \nu, \alpha}=O(1), \\
& \gamma_{\mu \nu, \alpha \beta}=O(1), \quad h_{\mu \nu, \alpha \beta}=O\left(\epsilon^{-1}\right) \text {, }
\end{aligned}
$$


are valid. Next, we expand the Ricci tensor in powers of $h$,

$$
R_{\mu \nu}(g)=R_{\mu \nu}^{(0)}+\varepsilon R_{\mu \nu}^{(1)}+\varepsilon^{2} R_{\mu \nu}^{(2)}+\varepsilon^{3} R_{\mu \nu}^{(3)}+\ldots,
$$

where

$$
\begin{aligned}
R_{\mu \nu}^{(0)}(\gamma) \equiv & R_{\mu \nu}(\gamma), \\
R_{\mu \nu}^{(1)}(\gamma, h) \equiv & \frac{1}{2} \gamma^{\rho \tau}\left(h_{\tau \mu ; \nu \rho}+h_{\tau \nu ; \mu \rho}-h_{\rho \tau ; \mu \nu}-h_{\mu \nu ; \rho \tau}\right), \\
R_{\mu \nu}^{(2)}(\gamma, h) \equiv & \frac{1}{2}\left[\frac{1}{2} h_{; \nu}^{\rho \tau} h_{\rho \tau ; \mu}+h^{\rho \tau}\left(h_{\tau \rho ; \mu \nu}+h_{\mu \nu ; \tau \rho}-h_{\tau \mu ; \nu \rho}\right.\right. \\
& \left.\quad-h_{\tau \nu ; \mu \rho}\right)+h_{\nu}^{\tau ; \rho}\left(h_{\tau \mu ; \rho}-h_{\rho \mu ; \tau}\right) \\
& \left.\quad-\left(h_{; \rho}^{\rho \tau}-\frac{1}{2} h^{; \tau}\right)\left(h_{\tau \mu ; \nu}+h_{\tau \nu ; \mu}-h_{\mu \nu ; \tau}\right)\right] . \\
R_{\mu \nu}^{(3)}(\gamma, h) \equiv & \frac{1}{4} h^{\sigma \tau} h_{\sigma \rho ; \mu} h_{\tau ; \nu}^{\rho}+\ldots .
\end{aligned}
$$

The semicolons denote covariant differentiation with respect to the background metric $\gamma_{\mu \nu}$, which is also used to raise or lower all indices. Considering relations (2), the orders of the terms (4) are

$$
R_{\mu \nu}^{(0)}=O(1), \varepsilon R_{\mu \nu}^{(1)}=O\left(\epsilon^{-1} \varepsilon\right), \varepsilon^{2} R_{\mu \nu}^{(2)}=O\left(\varepsilon^{2}\right), \varepsilon^{3} R_{\mu \nu}^{(3)}=O\left(\epsilon \varepsilon^{3}\right) .
$$

Two limiting cases thus arise naturally. For the standard linearization $(\varepsilon \ll 1, \epsilon=1)$ the dominant term of $R_{\mu \nu}(g)$ is $R_{\mu \nu}^{(0)}=O(1)$ which corresponds to the background $\gamma_{\mu \nu}$ [to find, e.g., a vacuum spacetime metric $g_{\mu \nu}$ we solve $\left.R_{\mu \nu}^{(0)}(\gamma)=0\right]$. Its first correction representing linearized (purely) gravitational waves is governed by

$$
R_{\mu \nu}^{(1)}(\gamma, h)=0
$$

which is a dynamical equation for perturbations $h_{\mu \nu}$ on the fixed background $\gamma_{\mu \nu}$. The next term $R_{\mu \nu}^{(2)}(\gamma, h)$ can then be used to define energy-momentum tensor of these gravitational waves, but the background metric is not assumed to be influenced by it. Improvements to this inconsistency can be obtained by iteration procedure. More rigorous but somewhat complicated solution to this problem was recently proposed by Efroimsky [35.

In the high-frequency approximation $(\epsilon \ll 1, \varepsilon=1)$ the dominant term is $R_{\mu \nu}^{(1)}=O\left(\epsilon^{-1}\right)$ which gives the wave equation (6) for the perturbations $h_{\mu \nu}$ on the curved background $\gamma_{\mu \nu}$ (considering a vacuum full metric $g_{\mu \nu}$ ). The two terms of the order $O(1)$, namely $R_{\mu \nu}^{(0)}$ and $R_{\mu \nu}^{(2)}$, are both used to give the Einstein equation for the background non-vacuum metric, which represents the essential influence of the high-frequency gravitational waves on the background. Of course, to obtain a consistent solution, one has to use both the wave equation and the Einstein equation for the background simultaneously.

\subsection{Linear approximation}

Interestingly, it follows that the wave equation for $h_{\mu \nu}$, which arises from the linear perturbation of the Ricci tensor in vacuum for both the above limiting cases $\varepsilon \ll 1, \epsilon=1$, and $\epsilon \ll 1, \varepsilon=1$, is the same equation (6). In analogy with the well-known theory of massless spin-2 fields in flat space 4] we wish to impose two TT gauge conditions,

$$
\begin{aligned}
h_{\mu \nu}{ }^{; \nu} & =0, \\
h^{\mu}{ }_{\mu} & =0 .
\end{aligned}
$$


In this gauge we arrive at the following wave equation

$$
\diamond h_{\mu \nu} \equiv h_{\mu \nu} ; \beta ; \beta-2 R_{\sigma \nu \mu \beta}^{(0)} h^{\beta \sigma}-R_{\mu \sigma}^{(0)} h^{\sigma}{ }_{\nu}-R_{\nu \sigma}^{(0)} h_{\mu}^{\sigma}=0,
$$

where the operator $\diamond$ is the generalization of flat-space d'Alembertian. Contracting (9) we obtain $\left(h^{\mu}{ }_{\mu}\right)_{; \beta}^{; \beta}=0$, so that the condition (8) is always consistent with (9). However, if we differentiate $\diamond h_{\mu \nu}$ and use equations (17), (2), we find that

$$
\begin{aligned}
& \left(\diamond h_{\mu \nu}\right)^{; \nu}=\left(R_{\nu \beta ; \mu}^{(0)}-2 R_{\mu \nu ; \beta}^{(0)}\right) h^{\nu \beta}, \quad \text { where } \\
& \left(\diamond h_{\mu \nu}\right)^{; \nu}=O\left(\epsilon^{-2}\right), \quad\left(R_{\nu \beta ; \mu}^{(0)}-2 R_{\mu \nu ; \beta}^{(0)}\right) h^{\nu \beta}=O(\epsilon) .
\end{aligned}
$$

Thus, in case of standard linearized waves $(\epsilon=1)$ there is an obvious inconsistency, except for backgrounds with a covariantly constant Ricci tensor (e.g., for the Einstein spaces). On the other hand, in the high-frequency limit $(\varepsilon=1)$, the inconsistency between (9) and (7) is extremely small (the left and the right sides of (10) differ by $\epsilon^{3}$ where $\epsilon \ll 1$ ). Moreover, for all background metrics of constant curvature the equations are fully consistent. This is an important advantage of the equation (9) containing also terms of non-dominant order (namely those proportional to the Riemann or Ricci tensors), if compared to other "simpler" wave equations (e.g., $h_{\mu \nu} ; \beta ; \beta=0$ ) for which the left and right sides of (10) generally differ by only two orders of magnitude.

\subsection{Nonlinear terms and the effective energy-momentum tensor}

Before considering the second-order terms we now extend the formalism to be applicable to a larger class of spacetimes with (possibly) non-vanishing energy-momentum tensor $T_{\mu \nu}$. Namely, $g_{\mu \nu}$ need not be a vacuum metric (as only considered in [1]) but it satisfies Einstein's equations

$$
R_{\mu \nu}(g)=8 \pi \tilde{T}_{\mu \nu}(g, \varphi)
$$

Here $\tilde{T}_{\mu \nu} \equiv T_{\mu \nu}-\frac{1}{2} g_{\mu \nu} T_{\beta}^{\beta}$, such that $T_{\mu \nu}(g, \varphi)$ depends on non-gravitational fields $\varphi$ and on the full metric $g_{\mu \nu}$ but it does not contain the derivatives of $g_{\mu \nu}$. Note that this admits as particular cases a presence of electromagnetic field, and also Einstein spaces when $\tilde{T}_{\mu \nu}=\frac{1}{8 \pi} \Lambda g_{\mu \nu}$. Under the assumptions (2) valid for the decomposition (11) we expand the equation (12) as

$$
\begin{aligned}
R_{\mu \nu}^{(0)}(\gamma)+\varepsilon R_{\mu \nu}^{(1)}(\gamma, h)+\varepsilon^{2} R_{\mu \nu}^{(2)}(\gamma, h)+\ldots= \\
\quad 8 \pi\left[\tilde{T}_{\mu \nu}^{(0)}(\gamma, \varphi)+\varepsilon \tilde{T}_{\mu \nu}^{(1)}(\gamma, h, \varphi)+\varepsilon^{2} \tilde{T}_{\mu \nu}^{(2)}(\gamma, h, \varphi)+\ldots\right]
\end{aligned}
$$

where $\tilde{T}_{\mu \nu}^{(0)}(\gamma, \varphi) \equiv \tilde{T}_{\mu \nu}(\gamma, \varphi)$, and the remaining terms on the right-hand side are linear and quadratic in $h$, respectively. The orders of magnitude of the terms in the expansion of the Ricci tensor have been described above, cf. (5). For the energy-momentum tensor one obtains

$$
\tilde{T}_{\mu \nu}^{(0)}=O(1), \quad \tilde{T}_{\mu \nu}^{(1)}=O(\epsilon), \quad \tilde{T}_{\mu \nu}^{(2)}=O\left(\epsilon^{2}\right) .
$$

For ordinary linearization we thus get the equations $R_{\mu \nu}^{(n)}=8 \pi \tilde{T}_{\mu \nu}^{(n)}$ in each order $n=0,1,2, \ldots$. For the high-frequency approximation we obtain from (13) in the leading order $O\left(\epsilon^{-1}\right)$ the equation (6) which is identical with the wave equation in the vacuum case. The second-order contributions, that are $O(1)$, represent an influence of the high-frequency gravitational waves and matter fields on the background,

$$
R_{\mu \nu}^{(0)}(\gamma)-8 \pi \tilde{T}_{\mu \nu}^{(0)}(\gamma, \varphi)=-R_{\mu \nu}^{(2)}(\gamma, h)
$$


This equation (which in case of a vacuum spacetime reduces to the Isaacson result) can be rewritten in the form of Einstein's equation for the background as

$$
G_{\mu \nu}^{(0)}(\gamma)-8 \pi T_{\mu \nu}^{(0)}(\gamma, \varphi)=-\left[R_{\mu \nu}^{(2)}(\gamma, h)-\frac{1}{2} \gamma_{\mu \nu} R^{(2)}(\gamma, h)\right] \equiv 8 \pi T_{\mu \nu}^{G W}
$$

This defines the effective energy-momentum tensor $T_{\mu \nu}^{G W}$ of high-frequency gravitational waves.

\subsection{Gravitational waves in the WKB approximation}

In the following we shall restrict ourselves to the Isaacson approximation $(\varepsilon=1, \epsilon \ll 1)$, i.e. on study of high-frequency gravitational waves on curved backgrounds. Inspired by the plane-wave solution in flat space, the form $h_{\mu \nu}=\mathcal{A} e_{\mu \nu} \exp (i \phi)$ of the solution is assumed. The amplitude $\mathcal{A}=O(\epsilon)$ is a slowly changing real function of position, the phase $\phi$ is a real function with a large first derivative but no larger derivatives beyond, and $e_{\mu \nu}$ is a normalized polarisation tensor field. The above assumption, introduced in [1], is called the WKB approximation, or the geometric optics limit [4]. The wave vector normal to surfaces of constant phase is $k_{\mu} \equiv \phi_{, \mu}$ and the orders of various relevant quantities are $R_{\mu \nu \gamma \delta}^{(0)}=O(1), \mathcal{A}_{, \mu}=O(\epsilon), k_{\mu}=O\left(\epsilon^{-1}\right)$, and $k_{\mu ; \nu}=O\left(\epsilon^{-1}\right)$. Substituting this into the conditions (7), (8), and the wave equation (9) we obtain, in the two highest orders which are gauge invariant,

$$
\begin{aligned}
& k^{\mu} k_{\mu}=0, \quad k^{\mu} e_{\mu \nu}=0, \quad k^{\alpha} e_{\mu \nu ; \alpha}=0, \\
& e^{\mu \nu} e_{\mu \nu}=1, \quad \gamma^{\mu \nu} e_{\mu \nu}=0, \quad\left(\mathcal{A}^{2} k^{\beta}\right)_{; \beta}=0 .
\end{aligned}
$$

These express that a beam of high-frequency gravitational waves propagate along rays which are null geodesics with tangent $k^{\mu}$, with parallelly transported polarization orthogonal to the rays. Moreover, using the WKB approximation of $T_{\mu \nu}^{G W}$ and the Brill-Hartle averaging procedure [6] (which guarantees the gauge invariance) Isaacson obtained for gravitational waves in the geometric optics limit the energy-momentum tensor [1]

$$
T_{\mu \nu}^{H F}=\frac{1}{64 \pi} \mathcal{A}^{2} k_{\mu} k_{\nu} .
$$

The energy-momentum tensor of high-frequency waves thus has the form of pure radiation. This fully agrees with results obtained by alternative techniques $[8,9,14$.

\section{Examples of high-frequency gravitational waves}

Now we present some explicit classes of high-frequency gravitational waves. These are obtained by the above described WKB approximation method considering specific families of background spacetimes with a privileged geometry.

\subsection{Non-expanding waves}

As the background we first consider the Kundt class [19,28] of non-expanding, twist-free spacetimes in the form 36

$$
\mathrm{d} s^{2}=F \mathrm{~d} u^{2}-2 \frac{Q^{2}}{P^{2}} \mathrm{~d} u \mathrm{~d} v+\frac{1}{P^{2}}\left(\mathrm{~d} x^{2}+\mathrm{d} y^{2}\right)
$$


with

$$
\begin{aligned}
& P=1+\frac{\alpha}{2}\left(x^{2}+y^{2}\right), \\
& Q=\left[1+\frac{\beta}{2}\left(x^{2}+y^{2}\right)\right] e+C_{1} x+C_{2} y, \\
& F=D \frac{Q^{2}}{P^{2}} v^{2}-\frac{\left(Q^{2}\right)_{, u}}{P^{2}} v-\frac{Q}{P} H,
\end{aligned}
$$

where $\alpha, \beta$, and $e$ are constants (without loss of generality $e=0$ or $e=1$ ), $C_{1}, C_{2}$ and $D$ are arbitrary functions of the retarded time $u$, and $H(x, y, u)$ is an arbitrary function of the spatial coordinates $x, y$, and of $u$.

In particular, these are Petrov type $N$ (or conformally flat) solutions of Einstein's equations with cosmological constant $\Lambda$ when $\alpha=-\beta=\frac{1}{6} \Lambda$ and $D=-2 \beta e+C_{1}^{2}+C_{2}^{2}$, see e.g. 37, 38, 39, 36. Such metrics represent exact pure gravitational waves propagating along principal null direction $\partial_{v}$ if $H$ satisfies the equation $P^{2}\left(H_{, x x}+H_{, y y}\right)+\frac{2}{3} \Lambda H=0$. However, in our treatment here the function $H$ does not describe exact gravitational waves but rather it characterizes the influence of high-frequency perturbations on the background metric, which is assumed to be initially given by (19), (20) with $H=0$.

We consider the phase of high-frequency gravitational waves given by $\phi=\phi(u)$, and we seek solution in the WKB form, namely

$$
h_{\mu \nu}=\mathcal{A} e_{\mu \nu} \exp (i \phi(u)),
$$

where the amplitude $\mathcal{A}$ and polarization tensor $e_{\mu \nu}$ are functions of the coordinates $\{u, v, x, y\}$. The corresponding wave vector is $k_{\mu}=(\dot{\phi}, 0,0,0)$, where the dot denotes differentiation with respect to $u$. Applying now all the equations (17) we obtain

$$
\begin{aligned}
\mathcal{A} & =\mathcal{A}(u, x, y) \\
e_{\mu \nu}^{+} & =\frac{1}{\sqrt{2} P^{2}}\left(\begin{array}{cccc}
0 & 0 & 0 & 0 \\
0 & 0 & 0 & 0 \\
0 & 0 & 1 & 0 \\
0 & 0 & 0 & -1
\end{array}\right), \\
e_{\mu \nu}^{\times} & =\frac{1}{\sqrt{2} P^{2}}\left(\begin{array}{llll}
0 & 0 & 0 & 0 \\
0 & 0 & 0 & 0 \\
0 & 0 & 0 & 1 \\
0 & 0 & 1 & 0
\end{array}\right) .
\end{aligned}
$$

The fact that the amplitude $\mathcal{A}$ is independent of the coordinate $v$ expresses non-expanding character of the waves. The special polarisation tensors, denoted as + and $\times$, are analogous to those used in the standard theory of linearized waves in flat space. A general polarisation is easily obtained by considering $e_{\mu \nu}=a e_{\mu \nu}^{+}+b e_{\mu \nu}^{\times}$, where $a^{2}(u, x, y)+b^{2}(u, x, y)=1$.

Using the Einstein tensor for the metric (19) with the cosmological term in equations (16) and (18), we determine the reaction of the background on the presence of the above high-frequency gravitational perturbations, namely

$$
\frac{Q}{P}\left[P^{2}\left(\frac{\partial^{2}}{\partial x^{2}}+\frac{\partial^{2}}{\partial y^{2}}\right)+\frac{2}{3} \Lambda\right] H(u, x, y)=\frac{1}{4} \mathcal{A}^{2}(u, x, y) \dot{\phi}^{2}
$$

Notice that $\mathcal{A}=O(\epsilon)$ and $\dot{\phi}=O\left(\epsilon^{-1}\right)$. Therefore, the influence of high-frequency gravitational waves on the background, represented by the function $H$, is of the order $O(1)$. These approximate 
solutions can obviously be compared to specific exact radiative vacuum solutions which are given by $H$ solving the field equation (23) with a vanishing right-hand side (when $\mathcal{A}=0$, i.e. highfrequency perturbation waves are absent).

The above waves are non-expanding with the wave-fronts $u=$ const. being two-dimensional spaces of constant curvature given by $\alpha=\frac{1}{6} \Lambda$, cf. (19). For $\Lambda=0$ these are plane-fronted waves, for $\Lambda>0$ they are spheres, and for $\Lambda<0$ hyperboloidal surfaces.

Another interesting subclass of the Kundt spacetimes of the form (19), (20) are explicit Petrov type $I I$ (or more special) metrics given by $\beta=\alpha, e=1, C=0$ and $D=2(\Lambda-\alpha)$, namely

$$
d s^{2}=\left[2(\Lambda-\alpha) v^{2}-H\right] \mathrm{d} u^{2}-2 \mathrm{~d} u \mathrm{~d} v+\frac{1}{P^{2}}\left(\mathrm{~d} x^{2}+\mathrm{d} y^{2}\right) .
$$

For $H=0$ these are electrovacuum solutions with the geometry of a direct product of two 2-spaces of constant curvature, in particular the Bertotti-Robinson, (anti-)Nariai or PlebańskiHacyan spaces [41,42,40,43, see e.g. 44,36. Considering again (21) we obtain the results (22) as in the previous case. However, the reaction of high-frequency waves on the background is now different. It is determined by the equations (16) and (18) with the energy-momentum tensor consisting of a cosmological term plus that of a uniform non-null electromagnetic field described by the complex self-dual Maxwell tensor $F^{\mu \nu}=4 \Phi_{1}\left(m^{[\mu} \bar{m}^{\nu]}-k^{[\mu} l^{\nu]}\right)$, where $\Phi_{1}=\sqrt{\alpha-\frac{\Lambda}{2}} e^{i c}$, $c=$ const., and $\mathbf{m}=P \partial_{\bar{\zeta}}, \mathbf{k}=\partial_{v}, \mathbf{l}=\frac{1}{2} F \partial_{v}+\partial_{u}$ form the null tetrad. Straightforward calculation gives

$$
P^{2}\left(\frac{\partial^{2}}{\partial x^{2}}+\frac{\partial^{2}}{\partial y^{2}}\right) H=\frac{1}{4} \mathcal{A}^{2}(u, x, y) \dot{\phi}^{2} .
$$

This result is analogous to the equation (23), but the present situation is now more complicated since the background spacetime is not vacuum but it contains electromagnetic field. (In fact, the term with the cosmological constant $\Lambda$ in (23) has been entirely compensated by this.) Therefore, we have to analyze the perturbation of the complete Einstein-Maxwell system, and its consistency.

The Einstein equations in the two highest orders (6) and (16) have already been solved. We will now demonstrate that the Maxwell equations are also satisfied in the high-frequency limit, namely $F^{\mu \nu}{ }_{\mid \nu}=O(\epsilon)$, where $\mid$ denotes the covariant derivative with respect to the full metric $g_{\mu \nu}$. Indeed, using antisymmetry of $F^{\mu \nu}$ we can write $F^{\mu \nu}{ }_{\mid \nu}=F^{\mu \nu}{ }_{, \nu}+\frac{1}{2} g^{\alpha \beta} g_{\alpha \beta, \nu} F^{\mu \nu}$. Considering (2) and the gauge condition (8) we obtain $g^{\alpha \beta} g_{\alpha \beta, \nu}=\gamma^{\alpha \beta} \gamma_{\alpha \beta, \nu}-h^{\alpha \beta} h_{\alpha \beta, \nu}+O\left(\epsilon^{2}\right)$ because $\gamma^{\alpha \beta} h_{\alpha \beta, \nu}-h^{\alpha \beta} \gamma_{\alpha \beta, \nu}=\left(h_{\beta}^{\beta}\right)_{; \nu}-2 h^{\alpha \beta} \gamma_{\alpha \beta ; \nu}=0$, so that

$$
F_{\mid \nu}^{\mu \nu}=F_{; \nu}^{\mu \nu}-\frac{1}{2} h^{\alpha \beta} h_{\alpha \beta, \nu} F^{\mu \nu}+O\left(\epsilon^{2}\right) .
$$

Consequently, if the original background represents an electrovacuum spacetime, $F^{\mu \nu}{ }_{; \nu}=0$, the Maxwell equations $F^{\mu \nu}{ }_{\nu}=O(\epsilon)$ for the full metric are satisfied in the dominant order $O(1)$ in the high-frequency limit $\epsilon \ll 1$. In addition, the field equations are valid also in the next order $O(\epsilon)$ for the new electromagnetic field

$$
\mathcal{F}^{\mu \nu}=\left(1+\frac{1}{4} h^{\alpha \beta} h_{\alpha \beta}\right) F^{\mu \nu},
$$

since using (26) we obtain $\mathcal{F}^{\mu \nu}{ }_{\mid \nu}=O\left(\epsilon^{2}\right)$. Starting from an electromagnetic field $F^{\mu \nu}$ satisfying $F^{\mu \nu}{ }_{; \nu}=0$ with respect to the background metric $\gamma_{\mu \nu}$, we can thus construct the electromagnetic field $\mathcal{F}^{\mu \nu}$ which satisfies the Maxwell equations $\mathcal{F}^{\mu \nu}{ }_{\mid \nu}=O\left(\epsilon^{2}\right)$ with respect to the full metric $g_{\mu \nu}$ in the presence of high-frequency gravitational waves. Both the Einstein and Maxwell equations 
are then satisfied in the two highest perturbative orders. Interestingly, these results hold for high-frequency perturbations of any "seed" electrovacuum background spacetimes.

In particular, if the backgrounds are direct product spacetimes (24) for $H=0$ with uniform non-null electromagnetic field $\Phi_{1}=$ const. then high-frequency gravitational waves (21), (22) introduce $H$ which is given by equation (25). According to (27), the electromagnetic field is perturbed by the term proportional to $h^{\alpha \beta} h_{\alpha \beta}=\mathcal{A}^{2} e^{2 i \phi}=O\left(\epsilon^{2}\right)$, see (17), namely

$$
\Phi_{1}^{g}=\Phi_{1}\left[1+\frac{1}{2} \mathcal{A}^{2}(u, x, y) e^{2 i \phi(u)}\right] .
$$

This remains non-null but it is no longer uniform. The full spacetime thus describes non-uniform, non-null electromagnetic field plus the null field of high-frequency gravitational waves.

\subsection{Cylindrical waves}

Next we consider the class of cylindrical Einstein-Rosen waves,

$$
d s^{2}=e^{2 \gamma-2 \psi}\left(-\mathrm{d} t^{2}+\mathrm{d} \rho^{2}\right)+e^{2 \psi} \mathrm{d} z^{2}+\rho^{2} e^{-2 \psi} \mathrm{d} \varphi^{2} .
$$

If the functions $\psi(t, \rho)$ and $\gamma(t, \rho)$ satisfy the corresponding field equations (see, e.g. [21], 28], or equations (33)-(35) below) these are exact radiative spacetimes of the Petrov type I. We conveniently define double null coordinates $u=\frac{1}{\sqrt{2}}(t-\rho)$ and $v=\frac{1}{\sqrt{2}}(t+\rho)$; in these coordinates $\{u, v, \varphi, z\}$ the metric takes the form

$$
d s^{2}=-2 e^{2 \gamma-2 \psi} \mathrm{d} u \mathrm{~d} v+e^{2 \psi} \mathrm{d} z^{2}+\frac{1}{2}(v-u)^{2} e^{-2 \psi} \mathrm{d} \varphi^{2} .
$$

We assume this to be the class of background universes into which we wish to introduce high-frequency gravitational waves. We assume again $\phi=\phi(u)$ implying the wave vector $k_{\mu}=(\dot{\phi}, 0,0,0)$, i.e. the WKB perturbation of the form (21). By applying all the conditions (17) we obtain

$$
\begin{aligned}
\mathcal{A} & =\frac{\mathcal{U}(u)}{\sqrt{v-u}}, \\
e_{\mu \nu}^{+} & =\frac{1}{\sqrt{2}} e^{-2 \psi}\left(\begin{array}{cccc}
0 & 0 & 0 & 0 \\
0 & 0 & 0 & 0 \\
0 & 0 & \frac{1}{2}(v-u)^{2} & 0 \\
0 & 0 & 0 & -e^{4 \psi}
\end{array}\right), \\
e_{\mu \nu}^{\times} & =\frac{1}{2}(v-u)\left(\begin{array}{cccc}
0 & 0 & 0 & 0 \\
0 & 0 & 0 & 0 \\
0 & 0 & 0 & 1 \\
0 & 0 & 1 & 0
\end{array}\right) ;
\end{aligned}
$$

notice that $v-u=\sqrt{2} \rho>0$. Thus the perturbative solution is given by

$$
h_{\mu \nu}=\frac{\mathcal{U}(u)}{\sqrt{v-u}} e_{\mu \nu} \exp (i \phi(u)) \text {. }
$$

The back-reaction on the background (contained in a specific modification of the metric functions $\gamma$ and $\psi$ ) is given by the following equations, cf. (18),

$$
\begin{aligned}
(v-u) \psi_{, u}^{2}+\gamma_{, u} & =-\frac{1}{16}(v-u) \mathcal{A}^{2} \dot{\phi}^{2}, \\
(v-u) \psi_{, v}^{2}-\gamma_{, v} & =0 \\
\psi_{, u v}-\frac{1}{2(v-u)}\left(\psi_{, v}-\psi_{, u}\right) & =0 .
\end{aligned}
$$


Interestingly, this set of equations is consistent: by differentiating equation (33) with respect to $v$, equation (34) with respect to $u$, and combining them, one obtains (35) provided the amplitude $\mathcal{A}(u, v)$ satisfies the equation

$$
\left((v-u) \mathcal{A}^{2}\right)_{, v}=0 \text {. }
$$

However, this is automatically satisfied for the amplitude (31). It is thus quite simple to introduce gravitational waves in the WKB approximation into the cylindrical spacetimes (30). If the functions $\gamma$ and $\psi$ representing the background are solutions of the vacuum equations [i.e. (33)(35) with a vanishing right-hand side of [33)] then for introducing high-frequency gravitational waves it is sufficient just to alter the function $\gamma$ as

$$
\gamma(u, v) \rightarrow \gamma(u, v)+\tilde{\gamma}(u)
$$

where

$$
\frac{\partial \tilde{\gamma}(u)}{\partial u}=-\frac{1}{16} \mathcal{U}^{2} \dot{\phi}^{2}
$$

In particular, when $\psi=0=\gamma$ the background (29) is a flat Minkowski space. By assuming nontrivial $\tilde{\gamma}$ we obtain Petrov type $N$ spacetime with high-frequency gravitational waves which have cylindrical wave-fronts. In a general case this perturbation is propagating in the background which is the Einstein-Rosen cylindrical wave of Petrov type I. The effect on background is given by the relation (38) where $\mathcal{U}(u)=O(\epsilon)$ is an arbitrary amplitude function.

The above described perturbations depend on the null "retarded" coordinate $u$ so that the high-frequency gravitational waves are outgoing ( $\rho$ is growing with $t$, on a fixed $u$ ). However, since the background metric (30) is invariant with respect to interchanging $u$ with $v$, it is straightforward to consider also ingoing perturbations by assuming the phase to depend on the "advanced coordinate" $v$, namely

$$
h_{\mu \nu}=\frac{\mathcal{V}(v)}{\sqrt{u-v}} e_{\mu \nu} \exp (i \phi(v))
$$

Then the term proportional to $\mathcal{A}^{2} \dot{\phi}^{2}$ will appear on the right-hand side of equation (34) instead of (33). This results in an interesting possibility to introduce ingoing high-frequency gravitational cylindrical waves into the background of outgoing Einstein-Rosen waves just by assuming $\tilde{\gamma}(v)$ in (37) such that

$$
\frac{\partial \tilde{\gamma}(v)}{\partial v}=+\frac{1}{16} \mathcal{V}^{2} \dot{\phi}^{2}
$$

or vice versa.

Moreover, all the above results can further be extended to a class of generalized EinsteinRosen (diagonal) metrics [29,45] which describe $G_{2}$ inhomogeneous cosmological models,

$$
d s^{2}=e^{2 \gamma-2 \psi}\left(-\mathrm{d} t^{2}+\mathrm{d} \rho^{2}\right)+e^{2 \psi} \mathrm{d} z^{2}+t^{2} e^{-2 \psi} \mathrm{d} \varphi^{2} .
$$

If the three-dimensional spacelike hypersurfaces are compact, the corresponding model is the famous Gowdy universe with the topology of three-torus [27,29. In the double null coordinates just one component of the metric is now different from (30), namely $g_{\varphi \varphi}=\frac{1}{2}(v+u)^{2} e^{-2 \psi(u, v)}$. The only modification of the above results (in the double null coordinates) consists of replacing the factor $(v-u)$ with $(v+u)$, and each derivative with respect to $u$ changing sign (e.g. $\gamma_{, u} \rightarrow-\gamma, u$ or $\left.\psi_{, u v} \rightarrow-\psi_{, u v}\right)$. High-frequency gravitational waves in inhomogeneous cosmologies of the form (41) can thus easily be constructed. 


\subsection{Expanding waves}

Finally, we assume that the background is an expanding Robinson-Trautman spacetime. The metric (generally of the Petrov type $I I$ ) in the standard coordinates has the form, see e.g. [22, 23, 28, 39,

$$
d s^{2}=-\left(K-2 r(\ln \mathcal{P})_{, u}-2 \frac{m}{r}-\frac{\Lambda}{3} r^{2}\right) \mathrm{d} u^{2}-2 \mathrm{~d} u \mathrm{~d} r+\frac{r^{2}}{\mathcal{P}^{2}}\left(\mathrm{~d} \eta^{2}+\mathrm{d} \xi^{2}\right),
$$

where $K=\Delta(\ln \mathcal{P}), \Delta \equiv \mathcal{P}^{2}\left(\frac{\partial^{2}}{\partial \eta^{2}}+\frac{\partial^{2}}{\partial \xi^{2}}\right)$, and $m(u)$. When $\mathcal{P}(u, \eta, \xi)$ satisfies the RobinsonTrautman equation $\Delta K+12 m(\ln \mathcal{P})_{, u}-4 m_{, u}=0$, the metric (42) is an exact vacuum solution of the Einstein equations.

In view of the existence of privileged congruence of null geodesics generated by $\partial_{r}$ we introduce the phase $\phi=\phi(u)$ and the wave vector $k_{\mu}=(\dot{\phi}, 0,0,0)$ of high-frequency gravitational waves. We again assume the WKB form (21) of the solution. Applying the equations (17) we obtain

$$
\begin{aligned}
\mathcal{A} & =\frac{1}{r} U(u, \eta, \xi) \\
e_{\mu \nu}^{+} & =\frac{1}{\sqrt{2}} \frac{r^{2}}{\mathcal{P}^{2}}\left(\begin{array}{llll}
0 & 0 & 0 & 0 \\
0 & 0 & 0 & 0 \\
0 & 0 & 1 & 0 \\
0 & 0 & 0 & -1
\end{array}\right), \\
e_{\mu \nu}^{\times} & =\frac{1}{\sqrt{2}} \frac{r^{2}}{\mathcal{P}^{2}}\left(\begin{array}{llll}
0 & 0 & 0 & 0 \\
0 & 0 & 0 & 0 \\
0 & 0 & 0 & 1 \\
0 & 0 & 1 & 0
\end{array}\right) .
\end{aligned}
$$

A general solution has the form $h_{\mu \nu}=r^{-1} U(u, \eta, \xi) e_{\mu \nu} \exp (i \phi(u))$, where $U(u, \eta, \xi)$ and $\phi(u)$ are arbitrary functions, and $e_{\mu \nu}=a e_{\mu \nu}^{+}+b e_{\mu \nu}^{\times}$with $a^{2}(u, \eta, \xi)+b^{2}(u, \eta, \xi)=1$. Introducing the amplitudes $U^{+}=a U, U^{\times}=b U$ for both polarizations, we can write the solution as

$$
h_{\mu \nu}=\frac{1}{r}\left[U^{+} e_{\mu \nu}^{+}+U^{\times} e_{\mu \nu}^{\times}\right] \exp (i \phi(u)) .
$$

If the wave-surfaces $r=$ const., $u=$ const. with the metric $d l^{2}=\mathcal{P}^{-2}\left(\mathrm{~d} \eta^{2}+\mathrm{d} \xi^{2}\right)$ are homeomorfic to $\mathcal{S}^{2}$, the waves can be interpreted as "spherical". In the asymptotic region $r \rightarrow \infty$ such solutions locally approach plane waves [16].

The reaction of the waves on background is determined by the equations (16) and (18) with $T_{\mu \nu}^{(0)}=-\frac{1}{8 \pi} \Lambda \gamma_{\mu \nu}$. From the only nontrivial component we immediately obtain the following equation

$$
-\frac{\partial m}{\partial u}+3 m(\ln \mathcal{P})_{, u}+\frac{1}{4} \Delta K=\frac{1}{16}\left[\left(U^{+}\right)^{2}+\left(U^{\times}\right)^{2}\right] \dot{\phi}^{2},
$$

where $m(u), \phi(u)$, whereas the remaining functions depend on coordinates $\{u, \eta, \xi\}$. Notice that this is independent of the cosmological constant $\Lambda$.

The expressions (44),(45) agree with results obtained by MacCallum and Taub [9] or recently by Hogan and Futamase [16] who used Burnett's technique [14]. Our results, which were derived by a straightforward approach, are slightly more general because they are not restricted to a constant frequency $\dot{\phi}=$ const. Particular subcase of the Vaidya metric has already been studied before by Isaacson [1] and elsewhere [8]. 


\section{Conclusions}

The Isaacson approach to study high-frequency perturbations of Einstein's equations was briefly reviewed and compared with the standard weak-field limit. In our contribution we generalized Isaacson's method to include non-vacuum spacetimes, in particular an electromagnetic field and/or a non-vanishing value of the cosmological constant $\Lambda$. Then we explicitly analyzed possible high-frequency gravitational waves in three large families of background universes, namely non-expanding spacetimes of the Kundt type, cylindrical Einstein-Rosen waves and related inhomogeneous cosmological models (such as the Gowdy universe), and the Robinson-Trautman expanding spacetimes. These backgrounds are of various Petrov types. For example, highfrequency gravitational waves can be introduced into electrovacuum conformally flat BertottiRobinson space, type $D$ Nariai and Plebański-Hacyan spaces, their type $N$ and type $I I$ generalizations, or into algebraically general Einstein-Rosen universes.

For construction of high-frequency gravitational perturbations we have employed the fact that all these spacetimes admit a non-twisting congruence of null geodesics. The corresponding tangent vectors $k^{\mu}$ are hypersurface orthogonal so that there exists a phase function $\phi$ which satisfies $\phi_{, \mu}=k_{\mu}$. The last equation in (17) can be put into the form $\frac{d}{d l}(\ln \mathcal{A})=-\Theta$, where $l$ is the affine parameter, and $\Theta=\frac{1}{2} k_{; \mu}^{\mu}$ is the expansion of the null congruence. This determines the behaviour of the amplitude $\mathcal{A}$ in the above spacetimes (22), (31), (43). The remaining equations (17) enables one to deduce the polarization tensors.

It has been also crucial that all the classes of spacetimes discussed admit exact solutions with the energy-momentum tensor of pure radiation, i.e., $G_{\mu \nu}-8 \pi T_{\mu \nu}=\frac{1}{8} \mathcal{A}^{2} k_{\mu} k_{\nu}$, where $T_{\mu \nu}$ is either constant (representing the cosmological constant) or it describes an electromagnetic field. The relation between high-frequency perturbations and exact radiative solutions of Einstein's equations in each class is thus natural. In particular, it is possible to determine explicitly the reaction of the background on the presence of high-frequency gravitational waves.

\section{Acknowledgements}

The work was supported in part by the grants GAČR 202/02/0735 and GAUK 166/2003 of the Czech Republic and the Charles University in Prague.

\section{References}

[1] Isaacson, R. A. (1968). Gravitational Radiation in the Limit of High Frequency I. and II., Phys. Rev. 166, 1263-1280.

[2] Einstein, A. (1916). Näherungsweise Integration der Feldgleichungen der Gravitation, Preuss. Akad. Wiss. Sitz. 1, 688-696.

[3] Einstein, A. (1918). Über Gravitationswellen, Preuss. Akad. Wiss. Sitz. 1, 154-167.

[4] Misner, C. W., Thorne, K. S., and Wheeler J. A. (1973). Gravitation (W. H. Freeman: San Francisco).

[5] Wheeler, J. A. (1962). Geometrodynamics (Academic Press: New York).

[6] Brill, D. R., and Hartle, J. B. (1964). Method of the Self-Consistent Field in General Relativity and its Application to the Gravitational Geon, Phys. Rev. 135, B271-B278. 
[7] Choquet-Bruhat, Y. (1968). Construction de solutions radiatives approchées des équations d'Einstein, Rend. Acc. Lincei 44, 345-348.

[8] Choquet-Bruhat, Y. (1968). Construction de solutions radiatives approchées des équations d'Einstein, Commun. Math. Phys. 12, 16-35.

[9] MacCallum, M. A. H., and Taub, A. H. (1973). The Averaged Lagrangian and HighFrequency Gravitational Waves, Commun. Math. Phys. 30, 153-169.

[10] Taub, A. H. (1980). High-Frequency Gravitational Waves, Two-Timing, and Avaraged Lagrangians, in General Relativity and Gravitation, Vol. 1, ed. A. Held (Plenum Press: New York), p. 539-555.

[11] Araujo, M. E. (1986). On the Assumptions Made in Treating the Gravitational Wave Problem by the High-Frequency Approximation, Gen. Rel. Grav. 18, 219-233.

[12] Araujo, M. E. (1989). Lagrangian Methods and Nonlinear High-Frequency Gravitational Waves, Gen. Rel. Grav. 21, 323-348.

[13] Elster, T. (1981). Propagation of High-Frequency Gravitational Waves in Vacuum: Nonlinear Effects, Gen. Rel. Grav. 13, 731-745.

[14] Burnett, G. A. (1989). The high-frequency limit in general relativity, J. Math. Phys. 30, 90-96.

[15] Taub, A. H. (1976). High Frequency Gravitational Radiation in Kerr-Schild Space-Times, Commun. Math. Phys. 47, 185-196.

[16] Hogan, P. A., and Futamase, T. (1993). Some high-frequency spherical gravity waves, J. Math. Phys. 34, 154-169.

[17] Brinkmann, H. W. (1925). On Riemann Spaces conformal to Euclidean spaces, Proc. Natl. Acad. Sci. U.S. 9, 1.

[18] Bondi, H., Pirani, F. A. E., and Robinson I. (1959). Gravitational waves in general relativity, III. Exact plane waves, Proc. Roy. Soc. Lond. A251, 519-533.

[19] Kundt, W. (1961). The Plane-fronted Gravitational Waves, Z. Phys. 163, 77-86.

[20] Ehlers, J., and Kundt, K. (1962). Exact solutions of the gravitational field equations, in Gravitation: an Introduction to Current Research, ed. L Witten (J. Wiley \& Sons: New York), p. 49-101.

[21] Einstein, A., and Rosen, N. (1937). On Gravitational Waves, Journ. Franklin. Inst. 223, 43-45.

[22] Robinson, I., and Trautman, A. (1960). Spherical Gravitational Waves, Phys. Rev. Lett. 4, 431-432.

[23] Robinson, I., and Trautman, A. (1962). Some spherical gravitational waves in general relativity, Proc. Roy. Soc. Lond. A265, 463-473.

[24] Bonnor, W. B., and Swaminarayan, N. S. (1964). An exact solution for uniformly accelerated particles in general relativity, Z. Phys. 177, 240-256. 
[25] Bičák, J. (1968). Gravitational radiation from uniformly accelerated particles in general relativity, Proc. Roy. Soc. A 302, 201-224.

[26] Kinnersley, W., and Walker, M. (1970). Uniformly accelerating charged mass in general relativity, Phys. Rev. D 2, 1359-1370.

[27] Gowdy, R. H. (1971). Gravitational waves in closed universes, Phys. Rev. Lett. 27, 826-829.

[28] Kramer, D., Stephani, H., MacCallum, M. A. H., and Herlt, E. (1980). Exact Solutions of Einstein's Field Equations (Cambridge University Press: Cambridge).

[29] Carmeli M., Charach Ch., and Malin S. (1981). Survey of cosmological models with gravitational, scalar and electromagnetic waves, Phys. Rep. 76, 79-156.

[30] Bičák, J., and Schmidt, B. G. (1989). Asymptotically flat radiative space-times with boostrotation symmetry: The general structure, Phys. Rev. D 40, 1827-1853.

[31] Bonnor, W. B., Griffiths, J. B., and MacCallum, M. A. H. (1994). Physical Interpretation of Vacuum Solutions of Einstein's Equations. Part II. Time-dependent Solutions, Gen. Rel. Grav. 26, 687-729.

[32] Bičák, J. (2000). Selected Solutions of Einstein's Field Equations: Their Role in General Relativity and Astrophysics, in Einstein's Field Equations and Their Physical Implications, ed. B G Schmidt (Springer Verlag: Berlin), p. 1-126.

[33] Feinstein, A. (1988). Late-time behavior of primordial gravitational waves in expanding universe, Gen. Rel. Grav. 20, 183-190.

[34] Bennett, C. L. et al. (2003). First year Wilkinson Microwave Anisotropy Probe (WMAP) observations: preliminary maps and basic results, Astrophys. J., to appear astro-ph/0302207.

[35] Efroimsky, M. (1992). Gravity waves in vacuum and in media, Class. Quantum Gravity 9, 2601-2614

[36] Podolský, J., and Ortaggio, M. (2003). Explicit Kundt type II and N solutions as gravitational waves in various type $D$ and $O$ universes, Class. Quantum Grav. 20, 1685-1701.

[37] Ozsváth, I., Robinson, I., and Rózga, K. (1985). Plane-fronted gravitational and electromagnetic waves in spaces with cosmological constant, J. Math. Phys. 26, 1755-1761.

[38] Siklos, S. T. C. (1985). Lobatchevski Plane Gravitational Waves, in Galaxies, Axisymmetric Systems and Relativity, ed. M A H MacCallum (Cambridge University Press: Cambridge), p. $247-274$.

[39] Bičák, J., and Podolský, J. (1999). Gravitational waves in vacuum spacetimes with cosmological constant. I. Classification and geometrical properties of nontwisting type $N$ solutions, J. Math. Phys. 40, 4495-4505.

[40] Nariai, H. (1951). On a new cosmological colution of Einstein's field equations of gravitation, Sci. Rep. Tôhoku Univ. 35, 62-67.

[41] Bertotti, B. (1959). Uniform electromagnetic field in the theory of general relativity, Phys. Rev. 116, 1331-1333. 
[42] Robinson, I. (1959). A Solution of Maxwell-Einstein Equations, Bull. Acad. Polon. 7, 351352.

[43] Plebański, J. F., and Hacyan, S. (1979). Some exceptional electrovac type D matrics with cosmological constant, J. Math. Phys. 20, 1004-1010.

[44] Ortaggio, M., and Podolský, J. (2002). Impulsive waves in electrovac direct product spacetimes with $\Lambda$, Class. Quantum Grav. 19, 5221-5227.

[45] Carmeli, M., and Charach, Ch. (1984). The Einstein-Rosen gravitational waves and cosmology, Found. Phys. 14, 963-986. 